\title{
Preoperative cinematic rendering of a sinus frontalis frontal bone fracture
}

\author{
Christian Ase1 $^{1 *}$, Michael Malek ${ }^{2}$ and Franz A Fellner ${ }^{1,3}$ \\ ${ }^{1}$ Central Radiology Institute, Kepler University Hospital, Medical Faculty of the Johannes Kepler University, Linz, Austria \\ ${ }^{2}$ Department of Cranio-Maxillofacial Surgery, Kepler University Hospital, Medical Faculty of the Johannes Kepler University, Linz, Austria \\ ${ }^{3}$ Medical Faculty of the Friedrich-Alexander-University of Erlangen-Nürnberg, Erlangen, Germany
}

\section{Background}

Radiologists, especially those working at medical facilities with trauma surgery and maxillofacial surgery units, frequently encounter complex fractures of the viscerocranium and neurocranium. Among the many challenges these entail is providing the surgeon with a detailed and comprehensive description of the extent of the fracture of the variously dislocated bone fragments in conjunction with the surgical correction and/or preoperative planning. To do so, an interdisciplinary image presentation is often helpful. This case report on a sinus frontalis frontal bone fracture demonstrates that Cinematic Rendering of multi-detector computed tomography (MDCT) data to produce photorealistic preoperative images provides a better visual overview than $2 \mathrm{D}$ reconstructions.

\section{Case report}

We report here on a 21-year-old patient who suffered a traumatic injury to his forehead. Clinical examination of the patient revealed a bilateral orbita hematoma; the result of palpation was overwhelming suspicion of a fracture. Due to these findings, the patient was sent for computed tomography imaging. A non-contrast enhanced MDCT with axial data acquisition and subsequent multiplanar reformation in coronal and sagittal orientation (Figure 1) was performed according to a standardized trauma protocol.

In the subsequent post-processing, the CT data set was visualized with 3D Cinematic Rendering, whereby both overview images of the entire skull from the front and various oblique and lateral views (Figure 2) as well as a coronal view and adequate sagittal section of the right half of the skull were produced (Figure 3).

The depressed fracture of the sinus frontalis frontal bone was repositioned and plated via bicoronal access. The subsequent postoperative conventional x-rays taken for control purposes revealed a good repositioning result as well as unobtrusive placement of the osteosynthesis material (Figure 4).

\section{Discussion}

Fractures of the sinus walls can entail complex fragmentations, in some cases due to bone thickness being only minimal in places. Frequently, the result is an opening of the sinus; occasionally, this is also closely associated with sensitive soft tissues, so that a concerted effort is made to plan surgery individually in order to achieve an optimal outcome [1,2]. From the radiological perspective, computed tomography imaging is usually performed to arrive at an exact diagnostic clarification of osseous injuries. Multiplanar reformed CT data sets allow for very precise analysis and understanding of fractures; nevertheless, in numerous cases, obtaining a detailed and comprehensive picture of complex fracture lines or dislocated fragments is possible only by simultaneously viewing images from several orientations as an overall picture. Producing 3D images via Cinematic Rendering of MDCT data sets yields a photorealistic depiction of facts and circumstances that are often very unclear and hard to grasp $[3,4]$. Therefore, in situations such as interdisciplinary demonstrations of findings or in conjunction with preoperative planning, this can be a very helpful tool to effectively convey information about a complex fracture situation.

\section{Conclusion}

The photorealistic, three-dimensional depiction of complex sinus fractures by means of Cinematic Rendering of MDCT data sets can be a useful preoperative imaging tool.

\section{Disclosure}

No conflicts of interest. No grants or financial support.

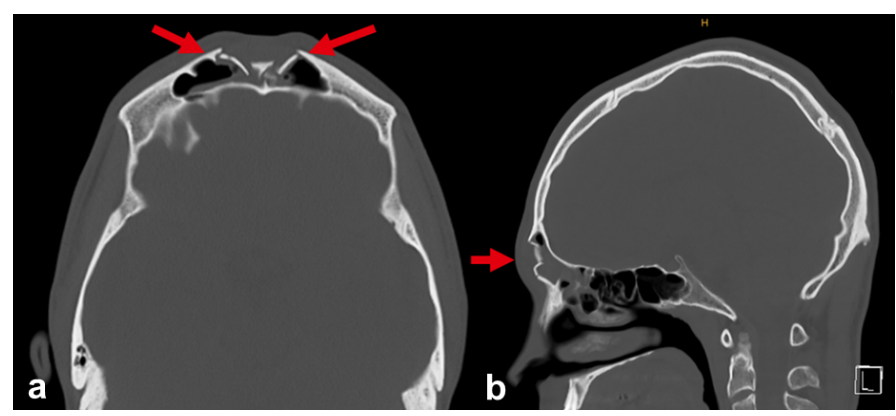

Figure 1. MDCT: Depressed fracture (arrow) of the Os frontale paramedian with opening of the frontal sinus cavity in an axial section (a) and a sagittal (b) multiplanar reconstruction.

Correspondence to: Christian Asel, Central Radiology Institute, Kepler University Hospital, Medical Faculty of the Johannes Kepler University, Linz, Austria, E-mail: christian.asel@kepleruniklinikum.at

Key words: trauma, fracture, computed tomography, preoperative imaging, 3D imaging, cinematic rendering, computed tomography, MDCT

Received: January 07, 2018; Accepted: January 24, 2018; Published: January 26, 2018 


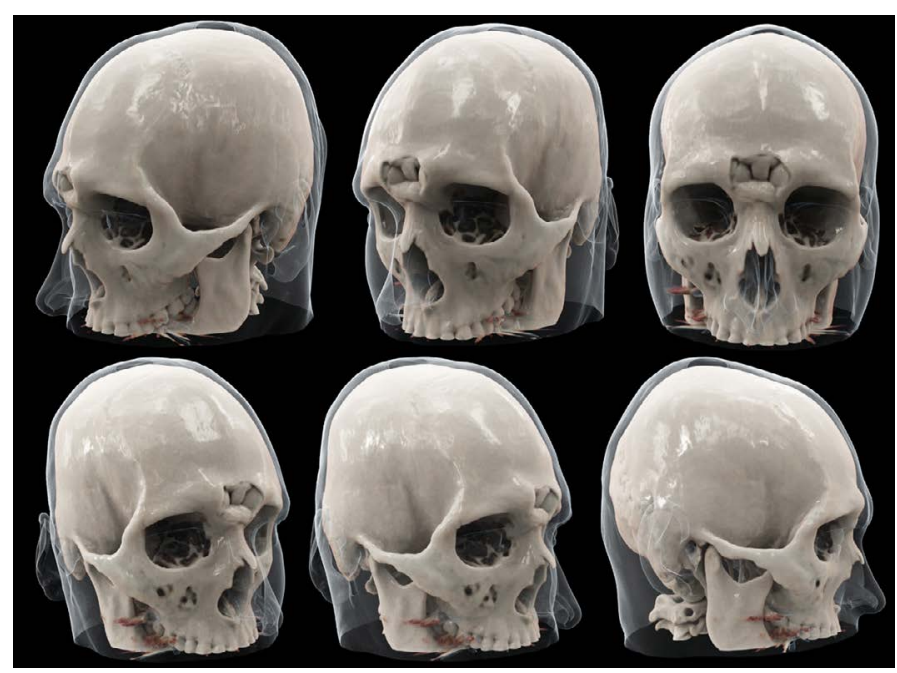

Figure 2. Various 3D Cinematic Rendering reconstructions of the depressed frontal fracture from the front and side.

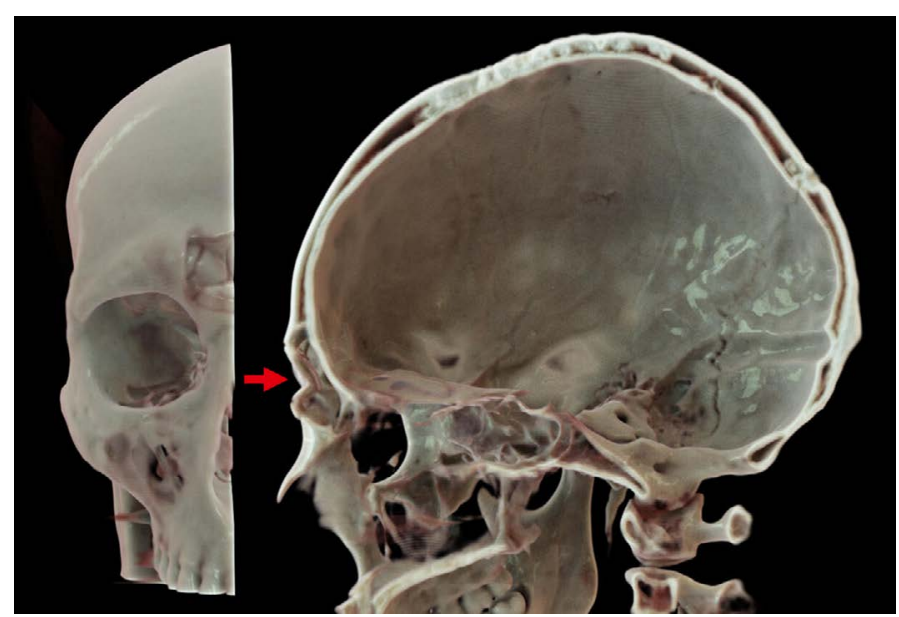

Figure 3. 3D Cinematic Rendering visualization of the right half of the skull in corona (left) and sagittal (right) views for additional depiction of the depressed fracture (arrow).

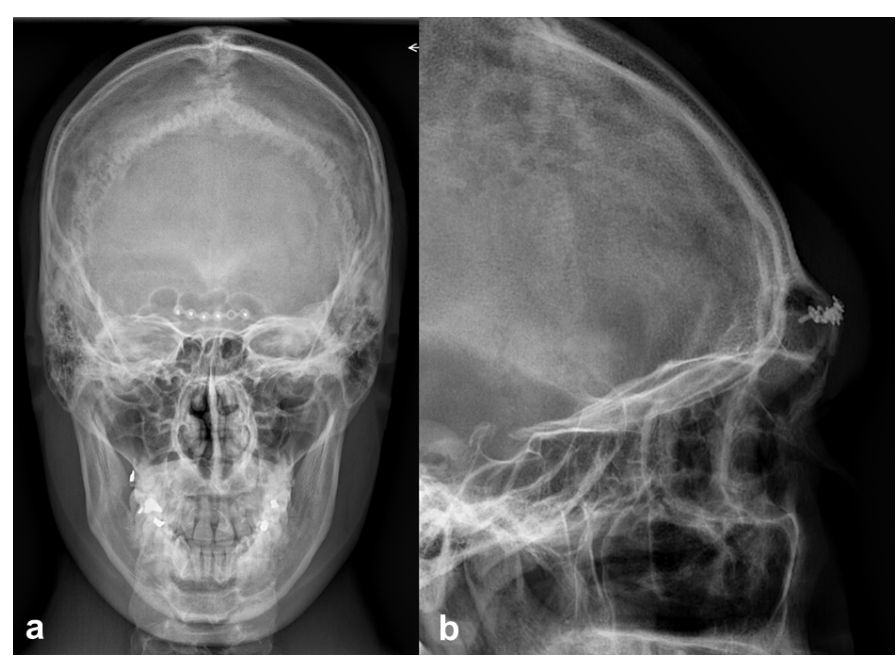

Figure 4. Postoperative x-rays taken anterior-posterior (a) and laterally (b) show a good repositioning result and correct situation of the osteosynthesis material.

\section{References}

1. Kim J, Choi H (2016) A review of subbrow approach in the management of noncomplicated anterior table frontal sinus fracture. Arch Craniofac Surg 17: 186-189. [Crossref]

2. Garg RK, Afifi AM, Gassner J, Hartman MJ, Leverson G, et al. (2015) A novel classification of frontal bone fractures: The prognostic significance of vertical fracture trajectory and skull base extension. J Plast Reconstr Aesthet Surg 68: 645-653. [Crossref]

3. Fellner FA (2016) Introducing cinematic rendering: A novel technique for postprocessing medical imaging data. J Biomed Sci Eng 09: 170-175.

4. Rowe SP, Fritz J, Fishman EK (2017) CT evaluation of musculoskeletal trauma: Initial experience with cinematic rendering. Emerg Radiol 25: 93-101. [Crossref]

Copyright: (C2018 Asel C. This is an open-access article distributed under the terms of the Creative Commons Attribution License, which permits unrestricted use, distribution, and reproduction in any medium, provided the original author and source are credited. 